\title{
Efficient colored silicon solar modules using integrated resonant dielectric nanoscatterers
}

Verena Neder, , Stefan L. Luxembourg, and, and Albert Polman

Citation: Appl. Phys. Lett. 111, 073902 (2017); doi: 10.1063/1.4986796

View online: http://dx.doi.org/10.1063/1.4986796

View Table of Contents: http://aip.scitation.org/toc/apl/111/7

Published by the American Institute of Physics

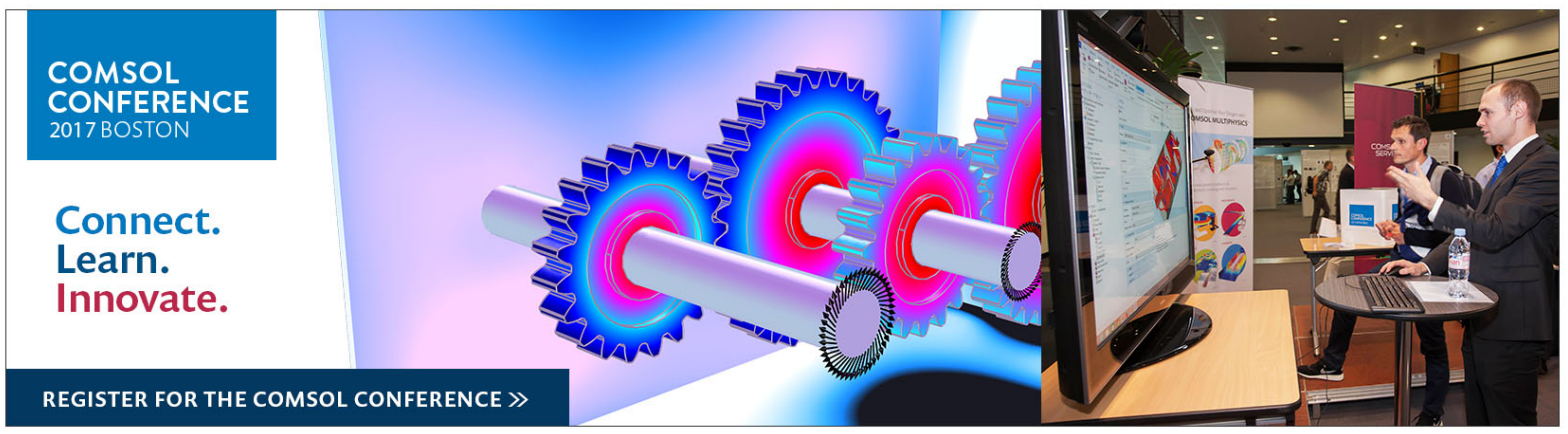




\title{
Efficient colored silicon solar modules using integrated resonant dielectric nanoscatterers
}

\author{
Verena Neder, ${ }^{1,2}$ Stefan L. Luxembourg, ${ }^{3}$ and Albert Polman ${ }^{2}$ \\ ${ }^{1}$ Institute of Physics, University of Amsterdam, Science Park 904, 1098 XH Amsterdam, The Netherlands \\ ${ }^{2}$ Center for Nanophotonics, AMOLF, Science Park 104, 1098 XG Amsterdam, The Netherlands \\ ${ }^{3}$ ECN Solar Energy, PO Box 1, 1755 ZG Petten, The Netherlands
}

(Received 6 June 2017; accepted 21 July 2017; published online 15 August 2017)

\begin{abstract}
We demonstrate photovoltaic modules with a bright green color based on silicon heterojunction solar cells integrated with arrays of light scattering dielectric nanoscatterers. Dense arrays of crystalline silicon nanocylinders, $100-120 \mathrm{~nm}$ wide, $240 \mathrm{~nm}$ tall, and $325 \mathrm{~nm}$ pitch, are made onto module cover slides using substrate-conformal soft-imprint lithography. Strong electric and magnetic dipolar Mie resonances with a narrow linewidth $(Q \sim 30)$ cause strong $(35 \%-40 \%)$ specular light scattering on resonance $(\sim 540 \mathrm{~nm})$. The green color is observed over a wide range of angles $\left(8^{\circ}-75^{\circ}\right)$. As the resonant nanoscatterers are transparent for the major fraction of the incident solar spectrum, the relative loss in short-circuit current is only $10 \%-11 \%$. The soft-imprinted nanopatterns can be applied on full-size solar modules and integrated with conventional module encapsulation. The dielectric Mie resonances can be controlled by geometry, opening up a road for designing efficient colorful or white building-integrated photovoltaics. Published by AIP Publishing.

[http://dx.doi.org/10.1063/1.4986796]
\end{abstract}

Photovoltaics (PV) can make a major contribution to the generation of renewable energy at a very large scale. In the past decades, PV research has focused on the development of PV materials and solar cell architectures with the aim to raise PV conversion efficiency and reduce manufacturing costs. For PV to be applied on a very large scale, it is essential that PV panels are well integrated into our built environment and landscape. A key property in this respect is the perceived color of PV panels. Typically, PV panels have a black or dark blue appearance that results from the textured surface of the solar cells in combination with a dielectric antireflection (AR) coating, designed to optimize the absorption of sunlight in the solar cell. However, for buildingintegrated applications, it would be beneficial to have PV panels in a variety of well-defined colors, while maintaining a high PV conversion efficiency.

So far, limited examples of colorful PV applications have been presented in the literature. Multilayer interference coatings can be applied to tailor the reflection spectrum but are expensive to make and show strong angle-dependent reflectivity, unless a large number of layers are used. ${ }^{1-3}$ Colored encapsulation of semitransparent thin-film silicon solar cells has been used to create solar panels with a red appearance for rooftop applications. ${ }^{4}$ However, this leads to a reduced efficiency due to light absorption by the polymer encapsulant. Similarly, assembled Ag nanostructures have recently been used to create colored solar panels due to the excitation of surface plasmon resonances, but at the price of Ohmic dissipation in the metal nanoparticles. ${ }^{5}$ Furthermore, the development of white solar panels was recently reported using an undisclosed geometry, resulting in a $40 \%$ loss in efficiency. ${ }^{6}$ So far, practical solutions for wide-angle colorful PV panels with high efficiency are scarce.

Dielectric nanostructures are known to have bright scattering colors due to the excitation of Mie resonances. The resonant spectrum can be tailored by particle shape and refractive index. ${ }^{7}$ If made from low-loss dielectrics, the Mie resonance can have a relatively narrow linewidth, resulting in a bright scattering spectrum with-depending on the used dielectric-negligible absorption losses. The angular distribution of the Mie scattered light can be tuned by tailoring the interplay of the electric and magnetic dipole and higher order modes. ${ }^{8}$ Several authors have demonstrated colorful examples of light scattering from arrays of Mie scatterers made on planar substrates. ${ }^{9-13}$

So far, the use of Mie scattering to create colorful PV panels has not been explored. Dielectric Mie scatterers have been applied onto the surface of solar cells as forward scattering geometries that enhance the light incoupling. ${ }^{14,15}$ Furthermore, Mie scatterers embedded in thin-film solar cells have been demonstrated to serve as light trapping geometries. ${ }^{16}$ Here, we introduce the use of dielectric Mie scatterers, integrated with the top cover layer of a solar panel, to selectively scatter a narrow band of the solar spectrum resulting in a bright green color. The unscattered blue and red/ infrared spectral ranges are efficiently coupled into the solar cell leading to a reduction in short circuit current density of only $10 \%$ (relative) compared to the uncolored reference.

Figure 1 shows the integrated Mie scattering designs that we developed. In Fig. 1(a), the dielectric scatterers are placed on top of a thin $1.2 \times 1.2 \mathrm{~cm}^{2}$ sapphire cover slide that is placed on top of a textured crystalline $\mathrm{Si}$ (c-Si) solar cell using index matching oil in between. In a second geometry [Fig. 1(b)], the Mie scatterers are placed on the bottom of the sapphire slide, facing the cell. The nanopatterned sapphire cover slides were placed on a front- and back-contacted Si heterojunction solar cell using index-matching fluid (Fluka 10976, $n=1.52$ ). The silicon heterojunction cells were made on $6^{\prime \prime} n$ type single-crystalline $\mathrm{Si}$ wafers and subsequently laserscribed to obtain $2 \times 2 \mathrm{~cm}^{2}$ cells. The surface of the cell was 
(a)

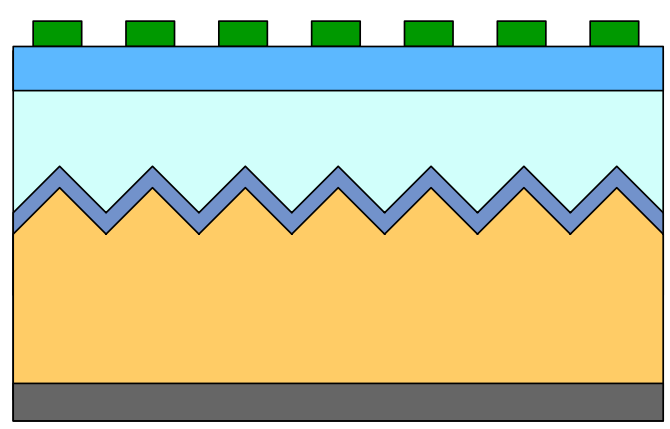

(c)

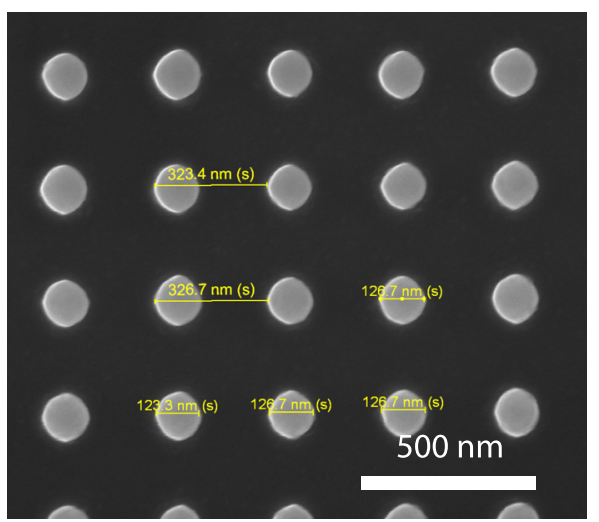

(b)

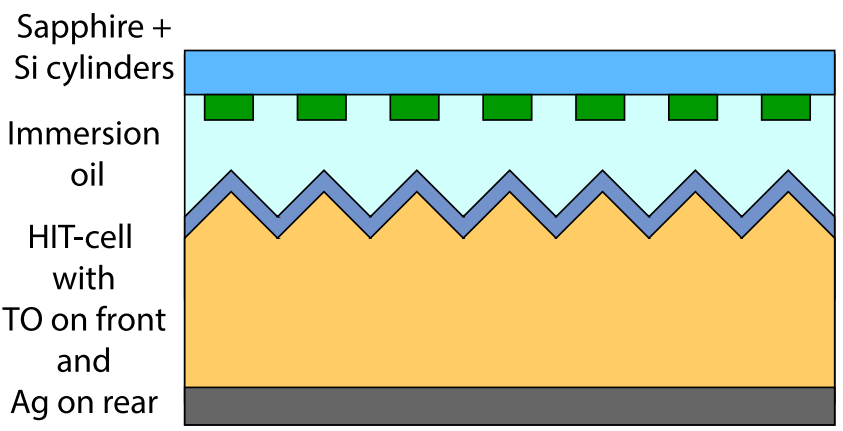

(d)

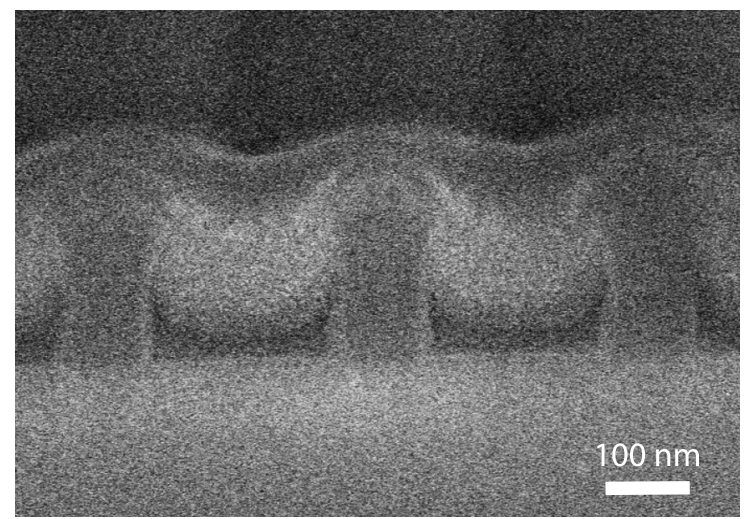

FIG. 1. Integrated Mie scattering designs for colored solar panels. (a) and (b) Schematic of silicon nanoscatterer arrays on top (a) and bottom (b) of a sapphire cover slide, integrated into a silicon heterojunction solar module using immersion oil. (c) Top-view SEM image of a square array of silicon nanoscatterers on a sapphire cover slide, made by SCIL. (d) SEM image of a cross section of the silicon nanoscatterers on sapphire made using focused ion beam milling.

textured using alkaline chemical etching, leading to a random pyramidal texture (base width $\sim 5 \mu \mathrm{m}$ ) and covered with an indium tin oxide (ITO) transparent conductor with an optimized thickness of $80 \mathrm{~nm}$ to act as an efficient AR coating $(n=2.05$ at $630 \mathrm{~nm})$. The use of index-matching oil enabled a flexible geometry to systematically compare the mini-modules (laminates) with and without the light scattering top layers. Sapphire was used to enable the use of crystalline Si Mie scatterers as crystalline silicon-on-sapphire substrates are commercially available. In practice, a similar geometry as proposed in Fig. 1 can be realized in a large-area solar panel by using a nanopatterned silica glass cover plate and an ethylene vinyl acetate (EVA) lamination layer, as commonly used in solar panels.

Silicon-on-sapphire substrates $(500 \mathrm{~nm} \mathrm{Si} \mathrm{on} 0.46 \mathrm{~mm}$ sapphire, MTI Corporation) were etched down to a thickness of $200 \mathrm{~nm}$ using reactive ion etching (RIE) in a $\mathrm{HBr}_{2}$ $(25 \mathrm{sccm})$ and $\mathrm{O}_{2}(2 \mathrm{sccm})$ plasma. Next, we used substrate conformal imprint lithography (SCIL) ${ }^{17}$ to fabricate the arrays of silicon cylinders. A 45-nm-thick silica sol-gel film was spin-coated and patterned using a SCIL stamp that was made from a pre-patterned silicon master, fabricated by electronbeam lithography. Two subsequent RIE etch steps were performed, first to break through the sol-gel pattern using $\mathrm{CHF}_{3}$ $(25 \mathrm{sccm})$ and $\operatorname{Ar}(25 \mathrm{sccm})$ and second to transfer the pattern into the silicon layer using $\mathrm{HBr}_{2} / \mathrm{O}_{2}$. The square array of silicon nanocylinders with a pitch of $325 \mathrm{~nm}$ is seen in the topview scanning electron microscopy (SEM) image in Fig. 1(c).
A cross-section of the structure is shown in Fig. 1(d). It shows that the particle diameter is slightly varying with height in the range $100-120 \mathrm{~nm}$; the particle height is $240 \mathrm{~nm}$. The size of the individual scatterers was chosen in order to create a visible reflection in green. By changing the size of the particles, the resonance can be shifted and different colors can be reflected. $^{12,13}$

Figure 2 (insets) depicts the light scattering spectra for the two geometries in Fig. 1. Measurements were performed with an integrating sphere in reflection configuration. A collimated beam from a supercontinuum light source was coupled in at an incidence angle of $8^{\circ}$. In this setup, all light that is scattered/reflected from the sample in the upper halfsphere is collected and sent to a spectrometer. In the insets, reference measurements for a bare Si heterojunction solar cell and the unpatterned module are also shown. The bare cell shows low reflection due to the combination of texture and optimized AR coating (interference minimum at $\sim 630 \mathrm{~nm}$ ). The unpatterned module has a reflectivity of $7 \%-12 \%$ as expected given the refractive index for sapphire. At long wavelengths, the nanopatterned module in (a) shows a lower reflectivity than the unpatterned reference due to an (effective-index) AR coating effect of the Si particles on top of the sapphire.

The scattering spectra of both integrated Mie scattering designs show a clear resonance (peak amplitude 35\%-40\%), due to the magnetic $(550 \mathrm{~nm})$ and electric $(528 \mathrm{~nm})$ dipole resonances. ${ }^{18,19}$ The resonance in Fig. 2(a) is slightly red- 
(a)

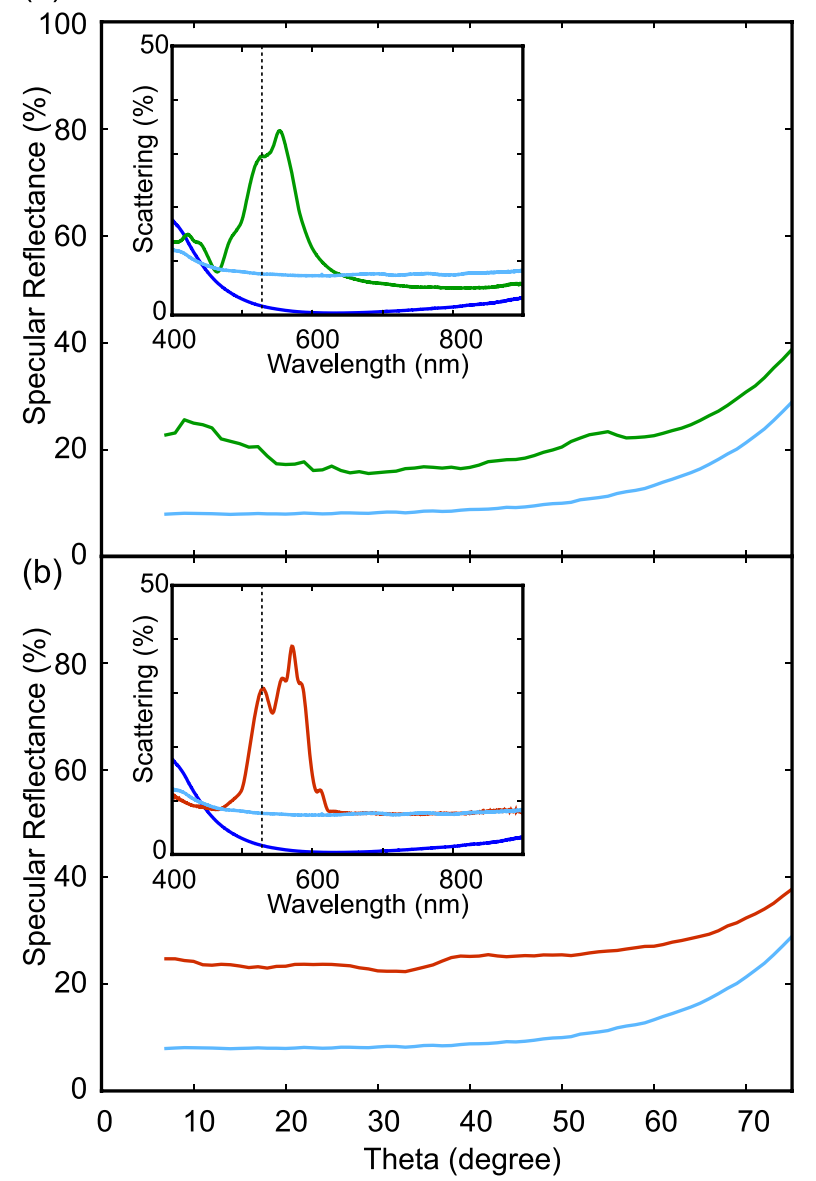

(c)

(d)
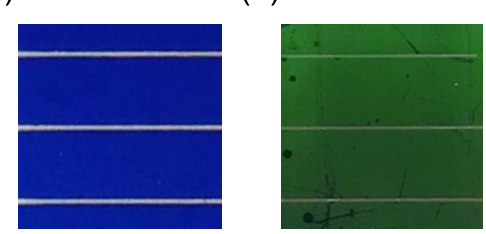

(e)

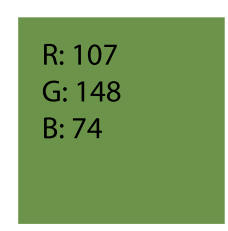

FIG. 2. Optical reflectance of nanopatterned Si heterojunction solar modules. Measured angle-dependent specular reflectance (averaging data for $s$ and $p$-polarized light) at the electric dipole resonance $(\lambda=528 \mathrm{~nm})$ of integrated design with nanoscatterers on top [(a), green] and bottom [(b), red] of the sapphire slide. Data for an unpatterned module are shown for reference (light blue). Insets: measured scattering efficiency of integrated design for top (a) and bottom (b) configurations. Data for an unpatterned module (light blue) and the bare Si heterojunction solar cell (dark blue) are also shown. Vertical black dashed lines at $\lambda=528 \mathrm{~nm}$ mark the electrical dipole resonance. Photographs $\left(0.5 \times 0.5 \mathrm{~cm}^{2}\right)$ of unpatterned modules (c) and nanopatterned module (d, bottom configuration), and (e) calculated visual color with the respective $\mathrm{RGB}$ values for the nanopatterned module.

shifted compared to that in Fig. 2(b) due to the higher index of the matching fluid. The linewidth of the scattering resonances (quality factor $Q \sim 30$ ) is mostly determined by (desired) radiation losses, in combination with small absorption losses. The main panels in Figs. 2(a) and 2(b) show the angle dependence of the specular reflectivity, measured at the peak of the electric dipole resonance. In both cases, the reflectance is strongly enhanced over the entire angular range from $8^{\circ}$ to $75^{\circ}$, when compared to the unpatterned module. This behavior is quite different than what is observed for simple multilayer interference coatings, from which the reflectivity is determined by Fabry-Perot interference which is strongly angle dependent at a given wavelength. In contrast, the localized nature of the Mie resonances used here enables strong light scattering within a narrow spectral band over a broad angular range. The insets in Fig. 2 also show that for off-resonant wavelengths the reflectivity does not deviate much from the reflectivity of the non-patterned reference. Figures 2(c) and 2(d) show the photographs of an unpatterned module and the nanopatterned module (bottom configuration), taken under diffuse daylight; a clear green color is observed. The visual color calculated from the measured reflection spectra [following the concept in Ref. 20, using the average midday light spectrum in Western Europe (D65) as illumination source] is shown in Fig. 2(e); it corresponds well to the color in the photograph of Fig. 2(d).

Figure 3(a) compares the measured external quantum efficiency (EQE) of the two nanopatterned modules, the unpatterned reference, and the bare Si solar cell. The activearea EQE spectrum was measured using slightly focused light ( $2 \mathrm{~mm}$ diameter) under normal incidence from a Xenon light source sent through a monochromator. The EQE of the bare Si heterojunction cell approaches $100 \%$ in the $600-900 \mathrm{~nm}$ spectral range. For the unpatterned geometry, the EQE is reduced by $7 \%-12 \%$ (relative) in agreement with the higher reflectivity observed in Fig. 2. The corresponding integrated short circuit current density $J_{\mathrm{sc}}$, derived from the EQE spectra, decreases from $39.5 \mathrm{~mA} / \mathrm{cm}^{2}$ to $36.8 \mathrm{~mA} / \mathrm{cm}^{2}$.

The EQE spectra for both integrated designs show strong dips $(25 \%-30 \%)$ at the resonant wavelengths, resulting from light scattering and absorption by the Si nanoscatterers. For the top configuration, the EQE is enhanced between 600 and $1000 \mathrm{~nm}$ in accordance with the reduced reflectivity [inset in Fig. 2(a)]. For both top and bottom configurations, the EQE is significantly reduced for wavelengths below the resonance, due to light absorption in the Si nanoscatterers. Correspondingly, $J_{\mathrm{sc}}$ drops to $33.2 \mathrm{~mA} / \mathrm{cm}^{2}$ (top configuration) and $32.7 \mathrm{~mA} / \mathrm{cm}^{2}$ (bottom configuration). Therefore, the (relative) reduction of the $J_{\mathrm{sc}}$ is $16 \%$ and $17 \%$ compared to the bare Si heterojunction cell and $10 \%$ and $11 \%$ compared to the unpatterned module with the sapphire slide.

The inset in Fig. 3(a) shows the current-voltage $(I-V)$ characteristics for the two patterned module configurations and the unpatterned reference. The $I-V$ curves were measured using an AM1.5G solar simulator (Newport Oriel Sol2A Class ABA), using a shadow mask to match the illuminated area to the size of the regular fabricated nanoparticle array $\left(0.7 \times 0.7 \mathrm{~cm}^{2}\right)$. The data are in full agreement with the trends observed in the EQE spectra, with the short-circuit current reduced due to the reduced light incoupling in the cells, and the bottom configuration showing slightly better performance than the top one. The $V_{\mathrm{oc}}$ values obtained from the $I-V$ measurements are strongly affected by the fact that the unmasked measurement area is much smaller than the cell area, creating an additional dark-current due to the unirradiated cell area (full-area 6 " cells from the same batch had a $V_{\text {oc }}$ of $724 \mathrm{mV}$ ). Obviously, the light incoupling effect of the nanopatterned geometry is best probed by changes in $J_{\mathrm{sc}}$, as these are a direct measure of changes in the light absorption. The conversion efficiency determined from the $I-V$ curves is $17.0 \%$ for the bare $\mathrm{Si}$ heterojunction cell, $15.9 \%$ for the unpatterned module and $13.7 \%$ and $12.9 \%$ for the 
(a)

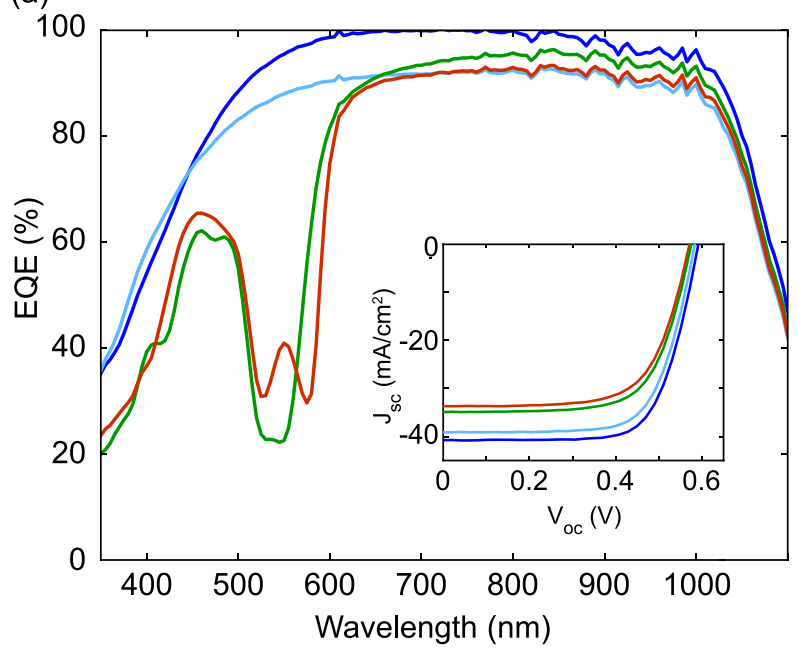

(b)

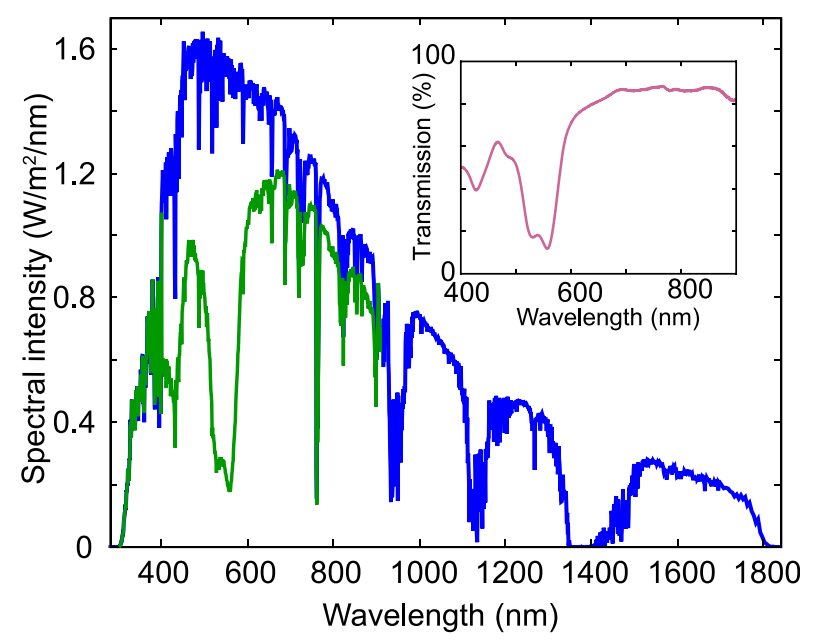

FIG. 3. Electrical measurements and solar spectrum. (a) Measured EQE of bare silicon heterojunction solar cell (dark blue), unpatterned module (light blue), and nanopatterned module in top (green) and bottom (red) configuration. Inset: $I$ - $V$ curves for the same cells. (b) AM1.5G solar spectrum (dark blue) and calculated modified spectrum after correction using the measured transmission spectrum (inset) of a nanopatterned sapphire slide (front configuration, green).

nanopatterned modules in front and bottom configuration, respectively. These efficiencies for the colored Si heterojunction solar modules correspond to a relative reduction in efficiency, compared to the unpatterned modules, of $14 \%$ and $19 \%$, respectively $[I-V$ measurements on a full-size cell under illumination levels corresponding to the current densities derived from the small-cell EQE spectra (using neutral density filters) provided effective (active-area) conversion efficiencies of $21.6 \%, 20.0 \%, 18.0 \%$ and $17.8 \%$ for the bare cell, unpatterned sapphire and front and bottom nanopatterned configurations, respectively (a relative change in efficiency of $10 \%$ and $11 \%$ )]. The lowest efficiency loss is thus achieved for the front patterned configuration, which is due to the (effective-index) AR coating effect of the frontnanopattern, as described above.

Finally, Fig. 3(b) shows the "effective" solar spectrum impinging on the Si heterojunction cell, taking into account the effect of the nanoscatterers. The standard AM1.5G solar spectrum is modified taking into account the transmission spectrum measured using an integrating sphere on a nanoparticle array on a bare sapphire slide [front configuration, inset in Fig. 3(b)]. The nanoscatterer array reduces the total integrated power from $1000 \mathrm{~W} / \mathrm{m}^{2}$ to $776 \mathrm{~W} / \mathrm{m}^{2}$. The ShockleyQueisser efficiency limit for a Si solar cell calculated using the modified spectrum is $32.8 \%$. The maximum power output for the nanopatterned cell derived from Fig. 3(b) is reduced by $24 \%$. This reduction is larger than that derived from the EQE data shown in Fig. 3(a) (16\%-17\%). We ascribe this difference to the fact that in the transmission measurements light is reflected from two sapphire interfaces while for the module cell geometry one sapphire interface is nearly index matched.

Our work has several advantages over earlier work on colored photovoltaics using plasmonic scattering. ${ }^{5}$ First of all, dielectric scattering is characterized by low absorption loss compared to the strong Ohmic dissipation in plasmonic scattering, as demonstrated by the relatively low loss in the short-circuit current observed above. Furthermore, when compared to the plasmonic resonances, geometric Mie resonances can be tuned over a larger spectral range while maintaining low loss, enabling a larger practical range of colors. The narrow linewidth observed for the Mie resonances also presents an advantage over colored photovoltaics based on absorbing dyes, which typically shows broadband absorption into the UV. The choice of the dielectric material for the resonators is determined by the need of a high index low-loss material. In this work, crystalline silicon was used because of its high dielectric constant and well known nanofabrication parameters.

Finally, we discuss the angular behavior of the integrated Mie scattering solar panel design presented here for practical applications. As shown in Fig. 2, the strong specular reflection at the resonance wavelength creates a clear green appearance of the cell for a broad range of incident angles. A further advanced design would scatter light from a single incident angle to a broad range of outgoing angles. Future work will focus on designing such surfaces.

In conclusion, we demonstrated efficient colored photovoltaic modules by the integration of dielectric nanoscatterer arrays in the front module layer using the soft-nanoimprint technology. The nanoscatterers show characteristic Mie resonances that create a well-defined scattering color. We present two designs, with Si nanoscatterers either on the top or on the bottom of the front module cover layer. A clear green appearance is observed over a wide range of angles for both configurations, with only $10 \%-11 \%$ loss in short circuit current and efficiency. The low-wavelength absorbance losses in the $\mathrm{Si}$ nanoscatterers used here can be avoided by using non-absorbing dielectrics such as $\mathrm{TiO}_{2} \cdot{ }^{11,21}$ The green solar modules presented here can find applications in buildingand landscape-integrated photovoltaics of many different kinds. The SCIL nano-imprint technique used is applicable to full-area $\left(15 \times 15 \mathrm{~cm}^{2}\right)$ solar cell patterning ${ }^{22}$ and can be readily scaled up to a roll-to-roll process that is applicable to full-size solar panels. The solar panel color can be changed by tuning the geometry of the dielectric nanoscatterers, which is readily done by adjusting the soft-imprint stamp. Furthermore, by using a combination of differently shaped 
Mie scatterers, photovoltaic modules with a white appearance can be designed as well.

We gratefully acknowledge Paula Bronsveld and her team for fabricating the Si heterojunction solar cells, Marc Verschuuren for helping with substrate conformal imprint lithography (SCIL), the assistance of Dimitry Lamers and Bob Drent for cleanroom support and Mark Knight for useful discussions. This work is part of the research program of the Netherlands Foundation for Scientific Research (NWO); it is also funded by the European Research Council.

${ }^{1}$ A. Schüler, C. Roecker, J.-L. Scartezzini, J. Boudaden, I. R. Videnovic, R. S.-C. Ho, and P. Oelhafen, Sol. Energy Mater. Sol. Cells 84, 241 (2004).

${ }^{2}$ A. Schüler, J. Boudaden, P. Oelhafen, E. De Chambrier, C. Roecker, and J. L. Scartezzini, Sol. Energy Mater. Sol. Cells 89, 219 (2005).

${ }^{3}$ M. Li, L. Zeng, Y. Chen, L. Zhuang, X. Wang, and H. Shen, Int. J. Photoenergy 2013, 352473.

${ }^{4}$ L. E. Perret-Aebi, P. Heinstein, V. Chapuis, S. Pélisset, C. Roecker, A. Schüler, K. Lumsden, Y. Leterrier, J. L. Scartezzini, J.-A. Manson, and C. Ballif, in Proceedings of the Conference on Cleantech for Sustainable Buildings (Loussane, 2011), p. 761.

${ }^{5}$ G. Peharz, K. Berger, B. Kubicek, M. Aichinger, M. Grobbauer, J. Gratzer, W. Nemitz, B. Großschädl, C. Auer, C. Prietl, W. Waldhauser, and G. C. Eder, Renewable Energy 109, 542 (2017).

${ }^{6}$ J. Escarre, H.-Y. Li, L. Sansonnens, F. Galliano, G. Cattaneo, P. Heinstein, S. Nicolay, J. Bailat, S. Eberhard, C. Ballif, and L.-E. PerretAebi, in 2015 IEEE 42nd Photovoltaics Specialists Conference (IEEE, 2015), pp. 1-2.
${ }^{7}$ J. Van De Groep and A. Polman, Opt. Express 21, 26285 (2013).

${ }^{8}$ R. Paniagua-Domínguez, Y. F. Yu, A. E. Miroshnichenko, L. A. Krivitsky, Y. H. Fu, V. Valuckas, L. Gonzaga, Y. T. Toh, A. Y. S. Kay, B. Luk'yanchuk, and A. I. Kuznetsov, Nat. Commun. 7, 10362 (2016).

${ }^{9}$ E.-H. Cho, H.-S. Kim, B.-H. Cheong, P. Oleg, W. Xianyua, J.-S. Sohn, D.J. Ma, H.-Y. Choi, N.-C. Park, and Y.-P. Park, Opt. Express 17, 8621 (2009).

${ }^{10}$ L. Cao, P. Fan, E. S. Barnard, A. M. Brown, and M. L. Brongersma, Nano Lett. 10, 2649 (2010).

${ }^{11}$ S. Sun, Z. Zhou, C. Zhang, Y. Gao, Z. Duan, S. Xiao, and Q. Song, ACS Nano 11, 4445 (2017).

${ }^{12}$ J. Proust, F. Bedu, B. Gallas, I. Ozerov, and N. Bonod, ACS Nano 10, 7761 (2016)

${ }^{13}$ Y. Kanamori, T. Ozaki, and K. Hane, Opt. Express 22, 25663 (2014).

${ }^{14}$ M. L. Brongersma, Y. Cui, and S. Fan, Nat. Mater. 13, 451 (2014).

${ }^{15}$ P. Spinelli, M. A. Verschuuren, and A. Polman, Nat. Commun. 3, 692 (2012).

${ }^{16}$ P. Spinelli and A. Polman, IEEE J. Photovoltaics 4, 554 (2014).

${ }^{17}$ M. V. Verschuuren, Substrate Conformal Imprint Lithography for Nanophotonic (Utrecht University, 2010).

${ }^{18}$ A. I. Kuznetsov, A. E. Miroshnichenko, Y. H. Fu, J. Zhang, and B. Luk'yanchuk, Sci. Rep. 2, 492 (2012).

${ }^{19}$ A. I. Kuznetsov, A. E. Miroshnichenko, M. L. Brongersma, Y. S. Kivshar, and B. Luk'yanchuk, Science 354, aag2472 (2016).

${ }^{20}$ J. Henrie, S. Kellis, S. M. Schultz, and A. Hawkins, Opt. Express 12, 1464 (2004).

${ }^{21}$ P. Spinelli, B. Macco, M. A. Verschuuren, W. M. M. Kessels, and A. Polman, Appl. Phys. Lett. 102, 233902 (2013).

${ }^{22}$ M. A. Verschuuren, M. Megens, J. F. Ni, H. van Sprang, and A. Polman, "Large area nanoimprint by substrate conformal imprint lithography (SCIL)," Adv. Opt. Tech. (published online). 